\title{
PERVIVENCIA DE LOS PAISAJES CERVANTINOS \\ EN EL CAMPO DE MONTIEL. \\ NOTAS A PARTIR DE UN TRABAJO DE CAMPO
}

\section{INTRODUCCIÓN}

En este trabajo se presentan los primeros resultados de una investigación que tuvo como objetivo inicial reconstruir la ruta de D. Quijote por el Campo de Montiel. Este proyecto se inspiraba en los trabajos, ya clásicos, destinados a tal fin por diversos autores ${ }^{1}$ y era sustentado también por la reconocida fidelidad de los escritos de Cervantes a la realidad geográfica ${ }^{2}$.

Este propósito nos condujo a realizar un conjunto de recorridos por el hipotético escenario de las aventuras de Don Quijote en busca de las actuales huellas de la organización territorial y los paisajes que el Campo de Montiel presentaba en la época de Cervantes.

Nuestra tarea se podría definir, quizás con algo de fantasía, como exploración, sin duda la vertiente más romántica de la investigación geográfica. Se trata de un trabajo apasionante, que hemos iniciado bajo la dirección de Justiniano Rodríguez Castillo, en el que, a partir de ese primer propósito de realizar una «arqueología imaginaria» del Quijote, estamos intentando reconocer la huella de la organización territorial del siglo XVI en el Campo de Montiel.

Cervantes, que tan ambiguo es en ocasiones en cuanto a referencias geográficas, insiste en fijar muy claramente al Campo de Montiel como teatro de buena parte de las aventuras de Don Qui-

\footnotetext{
1 Por ejemplo, JACACCI, A. F., El camino de Don Quijote por tierras de La Mancha, 1915; TERrero, J., "Las rutas de las tres salidas de D. Quijote», Anales Cervantinos, vol. VIII, pp. 1-49, 1960.

2 Caballero, F., "Conocimientos geográficos de Cervantes", Boletín de la Real Sociedad Geográfica, tomo XLVII, pp. 7-77, 1905.
} 
jote $^{3}$. Esa fijación nos induce a preguntarnos sobre qué le atrajo de nuestra comarca, sobre los paisajes o caracteres humanos que le inspiraron.

La curiosidad nos condujo a recorrer estos parajes para, al igual que hicieron Blázquez, Jacacci o Boscá ${ }^{4}$, relatar nuestras impresiones. Siguiendo la antigua red de caminos de la zona, hemos buscado - y en ocasiones encontrado- molinos, ventas y batanes. Más adelante exponemos los primeros resultados de esta investigación.

\section{UNAS NOTAS SOBRE LA VALORACIÓN GEOGRÁFICA DEL QUIJOTE}

El Quijote no es un libro de viajes. Sin embargo, la estructura, el hilo conductor sobre el que se fundamenta la obra, es, como en gran parte de las novelas de los siglos XVI y XVII, un viaje ${ }^{5}$. De ese carácter viajero se desprende la tentación de realizar una lectura geográfica de la gran obra cervantina.

Las numerosas investigaciones sobre la dimensión geográfica del Quijote pueden ser clasificadas, en una primera aproximación, en tres grupos:

- Las que destacan la pericia geográfica y la riqueza de conocimientos territoriales de Cervantes, derivados de su biografía viajera y su capacidad de observación. La precisión y elegancia que Cervantes emplea para verter sus conocimientos de geografía matemática y natural o para fijar los caracteres territoriales y humanos - con dos o tres epítetos- de diferentes regiones es reconocida con entusiasmo en trabajos de Foronda o Caballero ${ }^{6}$, entre otros. Quizás baste recordar el episodio del enfrentamiento entre dos huestes enemigas, encarnadas por sendos rebaños de ganado, para subrayar la habilidad y gracia de Cervantes para sintetizar sus vastos conocimientos geográficos.

3 Don Quijote. I. Prólogo; I. cap. 2; I. cap. 7; II. cap. 8. Se ha utilizado la edición de F. Rico. Barcelona, Instituto Cervantes-Crítica, 1998

4 Blázouez, A., "La Mancha en tiempos de Cervantes», Boletín de la Real Sociedad Geográfica, tomo XLVII, pp. 307-333, 1905; JACACCI, op. cit.; BosCÁ, E., "Tributo a Cervantes", Boletín de la Real Sociedad Española, tomo XVI, núm. 8, pp. 426433, 1916.

5 ISADO JIMÉnEZ, P. J., "Itinerarios y noticias de la novela picaresca en La Mancha (Ciudad Real)», Cuadernos de Estudios Manchegos, núm. 20, pp. 29-86, 1990; GARCÍA DE LA TORRE, M., "Realidad histórica y ficción literaria: el mundo de los caminos en Cervantes y su época», Anales Cervantinos, vol. XX, pp. 113-123, 1982.

6 Foronda y AgUilera, M., "Cervantes, viajero», Boletín de la Real Sociedad Geográfica, año V, núm. 6, pp. 419-484, 1880; CABALlERo, op.cit. 
- Las que tratan de reconstruir, mapa en mano, la ruta seguida por Don Quijote, intentando hacer coherentes la dimensión espacial y temporal de la obra, como si de un relato de viajes se tratara o, al menos, procurando identificar los lugares geográficos por los que anduvo el Caballero de la Triste Figura ${ }^{7}$.

- Las que se plantean de forma más general el marco geográfico del Quijote y la forma en que ha podido influir en Cervantes $^{8}$, o bien realizan interpretaciones literarias, humanistas o filosóficas del papel de los paisajes o elementos territoriales en la obra ${ }^{9}$.

A pesar de todo lo escrito, continúa el interés por realizar una lectura geográfica del Quijote; una lectura que debe ser realista por estas razones: en primer lugar, la obra, como novela que es, no está obligada a guardar coherencia cronológica y espacial; en segundo lugar, hay una deliberada ambigüedad territorial en la obra: aparecen escasas referencias a lugares conocidos en el recorrido del hidalgo y se aprecia poca precisión y parquedad en las descripciones paisajísticas, quizás porque a Cervantes le interesa más el mundo imaginario de Don Quijote que el prosaico que está recorriendo ${ }^{10}$. Por último, la combinación entre fantasía y realidad de la obra dificulta la tarea de reconocer los lugares que, sin duda, Cervantes conoció y en los que se inspiró.

Conscientes de todo ello pero, al tiempo, convencidos por la fijación de Cervantes en señalar a esta comarca como marco de las aventuras de Don Quijote, nos propusimos realizar una exploración literal, que no literaria, del territorio y comenzamos, como nuestro héroe, a caminar por el antiguo y conocido Campo de Montiel.

\section{El territorio del CAMPo de Montiel en tiempo de Cervantes}

Afortunadamente, contamos con excelentes investigaciones históricas que aportan información sobre aspectos demográficos, administrativos o económicos del campo de Montiel en la Edad

\footnotetext{
7 Agostini, E., y Gallego, R., Itinerarios y parajes cervatinos, 1936; TERRERo, op. cit., entre muchos otros.

8 Por ejemplo, Blázquez, op. cit.; García Pavón, M., «La Mancha que vio Cervantes», Anales Cervantinos, tomo IV, pp. 119-137, 1954.

9 Ver Garau Amengual, J., "El tratamiento del paisaje natural en el Quijote», Actas del II Coloquio Internacional de la Asociación de Cervantistas. Alcalá de Henares, Ed. Anthropos, 1991.

10 GARAU, op. cit.
} 
Moderna ${ }^{11}$. Sin embargo, la información estrictamente territorial - cartografiada o cartografiable- es más escasa.

Una fuente fundamental para adoptar una perspectiva geográfica o territorial es la constituida por las Relaciones Topográficas de Felipe $I I^{12}$, y su antecedente inmediato, la Descripción y cosmografía de España de Fernando Colón, realizada esta última en torno a los años veinte del siglo XVI. La aportación geográfica e histórica de estos documentos es extraordinaria y sorprende por su modernidad ${ }^{13}$. Apoyado en estas Relaciones, en los Diccionarios Geográficos de Madoz y Hervás y otras muchas fuentes de información, Corchado Soriano ${ }^{14}$ aborda la tarea de buscar la localización precisa de los elementos territoriales referidos en las mismas. Como resultado, el autor elabora un mapa histórico de gran interés (figura 1) que nos ha servido como base para nuestra exploración geográfica.

La singularidad del Campo de Montiel bajo el punto de vista histórico, que le hace fácilmente delimitable, se ve subrayada por los caracteres del medio físico. La comarca ha sido definida como una altiplanicie que se distingue claramente de su entorno, aunque las diferencias topográficas y geológicas internas dan lugar a una cierta diversidad paisajística. Se pueden apreciar contrastes entre las zonas más elevadas del Este y Noreste (donde se alcanza los 1.000 metros de altura), en las que predominan las formaciones naturales de encinar-sabinar, y las suaves ondulaciones modeladas sobre arcillas y margas triásicas hacia el Sudoeste, en las que sólo algunos cerros aislados superan los 850 metros de altura y se extienden los campos de cultivo, en ocasiones adehesados.

El medio físico explica buena parte de la organización territorial del Campo de Montiel en la Edad Moderna:

11 MAdRID MedinA, A., "El Campo de Montiel en la Edad Moderna», Cuadernos de Estudios Manchegos, núm. 9, II época, pp. 13-49, 1979; RUIz RoDRíGUEZ, J. I., Organización política y económica de la Orden de Santiago en el siglo XVII. Ciudad Real, Biblioteca de autores y temas manchegos, 1993.

12 Relaciones Topográficas de Felipe II. Ciudad Real, edición de VIÑas, C., y PAZ, R. Institutos Balmes de Sociología y Juan Sebastián Elcano de Geografía, CSIC, 1971.

13 Campos y Fernández de Sevilla, F. J., «Las relaciones topográficas de Felipe II: unas fuentes históricas monumentales sobre Castilla la Nueva en el siglo XVI», en La ciencia en el Monasterio del Escorial. San Lorenzo del Escorial, 1993; ARROYo IllERA, F., "Las relaciones geográficas y el conocimiento del territorio en tiempos de Felipe II», Estudios Geográficos, tomo LIX, núm. 231, pp. 169-199, 1998.

${ }_{14}$ Madoz, P., Diccionario Geográfico, Estadístico e Histórico de España y sus posesiones de ultramar, 1845; HERVÁs Y BUENDÍA, I., Diccionario Histórico-Geográfico de la provincia de Ciudad Real. Ciudad Real, Tip. del Hospicio Provincial, 1890; Corchado Soriano, Avance de un estudio geográfico-histórico del Campo de Montiel. Madrid, Patronato José María Cuadrado del Consejo Superior de Investigaciones Científicas, 1971. 
- Su situación geográfica en el contacto entre la Meseta y Andalucía hace que sea definido como zona de paso y encrucijada de caminos ya en la antigüedad, desde Jaén y Granada fundamentalmente, a través del puerto de San Esteban. Testimonios históricos de viajeros ilustres lo corroboran. Como se observa en la figura 1, la red de vías romanas, caminos reales y cañadas se abre en forma de abanico a partir del paso de Sierra Morena hacia distintas direcciones de la Meseta y Levante. Varios de los municipios que jalonan estos caminos - como Villamanrique o Puebla del Príncipe- son descritos en las Relaciones Topográficas de Felipe II como «muy pasajeros».

- La localización de los núcleos de población está relacionada también con necesidades históricas y las condiciones del medio físico. Así, las funciones defensivas justifican la ubicación de algunos pueblos en cerros-testigo (Alhambra, Montiel, Albaladejo). Por supuesto, el sistema de poblamiento está también determinado por el entramado viario supralocal. De hecho, Montiel y Alhambra, dos de los núcleos más importantes desde la Edad Media, estaban situado en las vías romanas que cruzaban el Campo de Montiel.

- La red hidrográfica constituye un elemento organizador del territorio de capital importancia. Además de algunos regadíos, la energía hidráulica constituye la base de la actividad fabril de la zona, que se concreta en la existencia de numerosos molinos harineros y batanes, industrias que se alinean a lo largo de los ríos más importantes como el Jabalón o el Guadalén.

Ángela Madrid ${ }^{15}$ nos habla de una economía local en la Edad Moderna que se basa, como en toda Castilla, en el binomio agricultura-ganadería, aunque con un predominio de la primera ante la progresiva decadencia de la segunda. La introducción del cultivo del viñedo constituye una novedad interesante en la época. La producción doméstica se complementa con la caza, la pesca y la leña, en un sistema típico de autoabastecimiento. La impronta de la red viaria supralocal en la zona justifica la relativa importancia de los intercambios comerciales, ferias y mercados, especialmente con Andalucía.

Por último, las Relaciones de Felipe II nos ofrecen la imagen de un territorio que ya había sufrido crisis: Los encuestados hacen advertencias sobre el agotamiento de las reservas de leña en algu-

\footnotetext{
15 Op. cit.
} 
nos municipios; por otra parte, se encuentran numerosas referencias a aldeas y caseríos despoblados ya en el siglo XVI.

\section{4. las huellas de los paisajes cervantinos en el Campo de MONTIEL}

Como señalábamos en la introducción, nuestro primer objetivo consistió en buscar la localización geográfica de los elementos territoriales mencionados por Cervantes en el recorrido de su héroe por el Campo de Montiel. Para ello, contábamos con excelentes trabajos sobre los tiempos y lugares en el Quijote ${ }^{16}$. Más que la realización de un mero inventario de parajes y monumentos, pretendíamos explicar la lógica de su ubicación, interpretarla a la luz de la organización territorial e, incluso, desentrañar el origen de su denominación. Estamos todavía en una primera fase de ese proyecto.

Abordar la tarea, en las puertas del siglo XXI, de reconocer las huellas que el siglo XVI dejó en la comarca podría parecer absurdo. Sin embargo, partimos de la hipótesis de que, al tratarse de una zona deprimida, pocos cambios paisajísticos se han producido. Ya A. Blázquez ${ }^{17}$ realizó una comparación de las relaciones descriptivas de 1575 con los mapas topográficos de la zona, comprobando que ni bosques, ni cultivos, ni molinos habían sufrido cambios importantes y que lo único que parecía renovarse eran las generaciones de agricultores que labraban aquellas tierras. En nuestra labor de reconocimiento, partimos de la antigua red de caminos en la zona entendiéndola como el elemento vertebrador del territorio en el que se apoyaban núcleos de población, ventas, puentes y fábricas. En esta primera fase del trabajo, nos hemos centrado en el sector sur-sureste del Campo de Montiel, porque, al ser zona de paso natural, presenta mayor densidad de caminos e intensidad de ocupación humana.

El procedimiento seguido en el trabajo es el siguiente:

- Búsqueda de referencias a elementos territoriales (caminos, poblaciones, parajes, etc.) en el texto del Quijote así como en diversa documentación literaria ${ }^{18}$ e histórica ${ }^{19}$ y en trabajos de síntesis sobre la comarca ${ }^{20}$.

16 CASASAYAS, J. M., «Lugares y tiempos en el Quijote», F. Rico ed. Don Quijote de La Mancha, volumen complementario pp. 911-933. Barcelona, Instituto Cervantes-Crítica, 1998.

17 BLÁzQUEZ, op. cit.

18 Las obras sobre la dimensión geográfica del Quijote ya mencionadas.

19 VIÑAS Y PAZ, op. cit.; MADOZ, op. cit.; HERVÁs, op. cit.

20 Planchuelo Portales, G., Estudio del Alto Guadiana y de la altiplanicie del Campo de Montiel. Instituto de Estudios Manchegos del CSIC, 1954 (reeditado en 
- Localización de tales componentes en cartografía actual ${ }^{21}$. En esta tarea, la toponimia ha constituido una referencia básica, además de una fuente inagotable de curiosidades y sorpresas. Como bien señala Blázquez ${ }^{22}$, «de cada diez fincas rústicas, ocho conservan los nombres primitivos, habiendo molino nuevo desde hace más de trescientos años...».

En la descripción que ahora sigue, hemos seleccionado los elementos localizados con mayores resonancias cervantinas y los presentamos agrupados según funciones territoriales:

En el Quijote, como en otros muchos de sus escritos, Cervantes nos ofrece una magnífica perspectiva del mundo de las ventas y caminos. Respecto a estas actividades camineras, los antiguos mapas topográficos y su toponimia nos permiten reconocer sin mayores problemas algunos sectores de vías romanas, cañadas y los caminos reales recogidos en los repertorios de caminos del siglo XVI (algunos con nombres tan evocadores como Camino Real de la Plata o Vía de Aníbal).

Relacionados con esta antigua red viaria, hemos localizado puentes, como el romano de tres arcos sobre el río Jabalón, en el camino real de Villanueva de los Infantes a Granada, y, sobre todo, ventas. Aunque se podrían citar varias, quizás la más importante de todas sea la Venta Nueva, junto al haz de caminos que penetra por el Sur en Sierra Morena. La Venta Nueva aparece referida en otras novelas de la época ${ }^{23}$ y también había sido citada en la literatura de viajes de los tiempos de Cervantes. Hemos podido reconocer personalmente que se conserva, tanto en su estructura interior (inmenso hogar con poyo interior para el asiento de los arrieros y dos habitaciones en alto para viajeros «de calidad»),como exterior, con el escudo de Gómez Suárez de Figueroa, quien la reconstruyó en el siglo XVII (figura 2).

Un segundo elemento territorial presente en la obra de Cervantes es el constituido por fábricas y molinos. Como dependientes de la energía hidráulica que son, estas industrias se sitúan a lo largo de los principales ejes fluviales de la comarca. A. Madrid ${ }^{24}$ contabiliza en torno a noventa molinos harineros a partir de las Relaciones de

1992 por la Biblioteca de autores y temas manchegos de la Diputación Provincial de Ciudad Real); CORCHADO SORIANO, op. cit.

21 Fundamentalmente en los Mapas Topográficos Nacionales a escala 1:50.000 correspondientes a las ediciones realizadas en torno a 1880 y 1950.

22 Op. cit.

23 ISADO JiMÉNEZ, op. cit.

${ }^{24}$ Op. cit. 
Felipe II. Corchado ${ }^{25}$ identifica, a partir de la primera edición del Mapa Topográfico Nacional, la localización de setenta y cuatro de esos molinos. Los batanes destinados al trabajo de los tejidos de lana, tan presentes en las aventuras de Don Quijote, también están representados en la comarca (Corchado identifica tres). En nuestros recorridos por el Campo de Montiel hemos localizado las ruinas de algunos molinos, como el del Marqués en el río Guadalén, y los bien conservados batanes, con gran parte de su maquinaria de madera, de Villanueva de la Fuente (figura 3).

Aunque en el siglo XVI ya se hallasen en decadencia, dada su inoperancia, hay que señalar la existencia de un conjunto de fortalezas y castillos que, sin duda, estuvieron en la mente de Cervantes cuando nos narraba las fantasías guerreras de D. Quijote. Hervás ${ }^{26}$ nos comenta la existencia, en el siglo XIII, de treinta y dos castillos distribuidos por el Campo de Montiel; muchos de ellos tuvieron alternativamente uso árabe y cristiano, según las fases de la Reconquista. Quizás el mejor conservado sea el castillo de Sant Yagüe de Montizón, del que fue comendador D. Jorge Manrique. A pesar de su ruina, todavía hoy su grado de conservación es estimable, lo que, unido a su atractivo emplazamiento en un estrecho del río Guadalén, hacen aconsejable su visita (figura 4).

Por último, se debe hacer una referencia a los lugares citados expresa e inequívocamente por Cervantes en su Quijote. El autor, que tan deliberadamente ambiguo en las referencias geográficas es en otros pasajes del libro, se muestra minucioso y preciso cuando sitúa a su personaje en el entorno de Montesinos, Ruidera y Rochafrida. Su descripción de las peculiaridades hidrogeológicas del Guadiana y del legendario origen de las lagunas de Ruidera, aunando realismo y fantasía, es de una belleza excepcional. La cueva de Montesinos, las lagunas, y el castillo de Rochafrida (figuras 5, 6 y 7), cuyo romance es recogido en las Relaciones Topográficas de Felipe II, son todos lugares que, sin duda, atrajeron especialmente la atención de nuestro autor ${ }^{27}$.

En conclusión, Cervantes conoció y visitó el Campo de Montiel. $\mathrm{Su}$ insistencia en citarlo como teatro de gran parte de las aventuras de D. Quijote nos conduce a preguntarnos por el atractivo de sus parajes y caracteres humanos para el autor. Con la intención de reconocer aquellos lugares, hemos comenzado a recorrer la co-

25 Op. cit.

26 Op. cit.

27 Tal como indica BlázQuez, op. cit., con seguridad Cervantes escuchó la leyenda de Rochafría y Montesinos directamente de los vecinos de La Solana, lugar en el que se conservaba la leyenda. 
marca. También hemos tenido el objetivo de dar a conocer su valor como un patrimonio cultural poco conocido y que puede ser, como lo fue nuestro héroe, universal.

MARÍA ÁNGELES DíAZ MUÑoz

Departamento de Geografía Universidad de Alcalá de Henares 


\section{APÉNDICE}

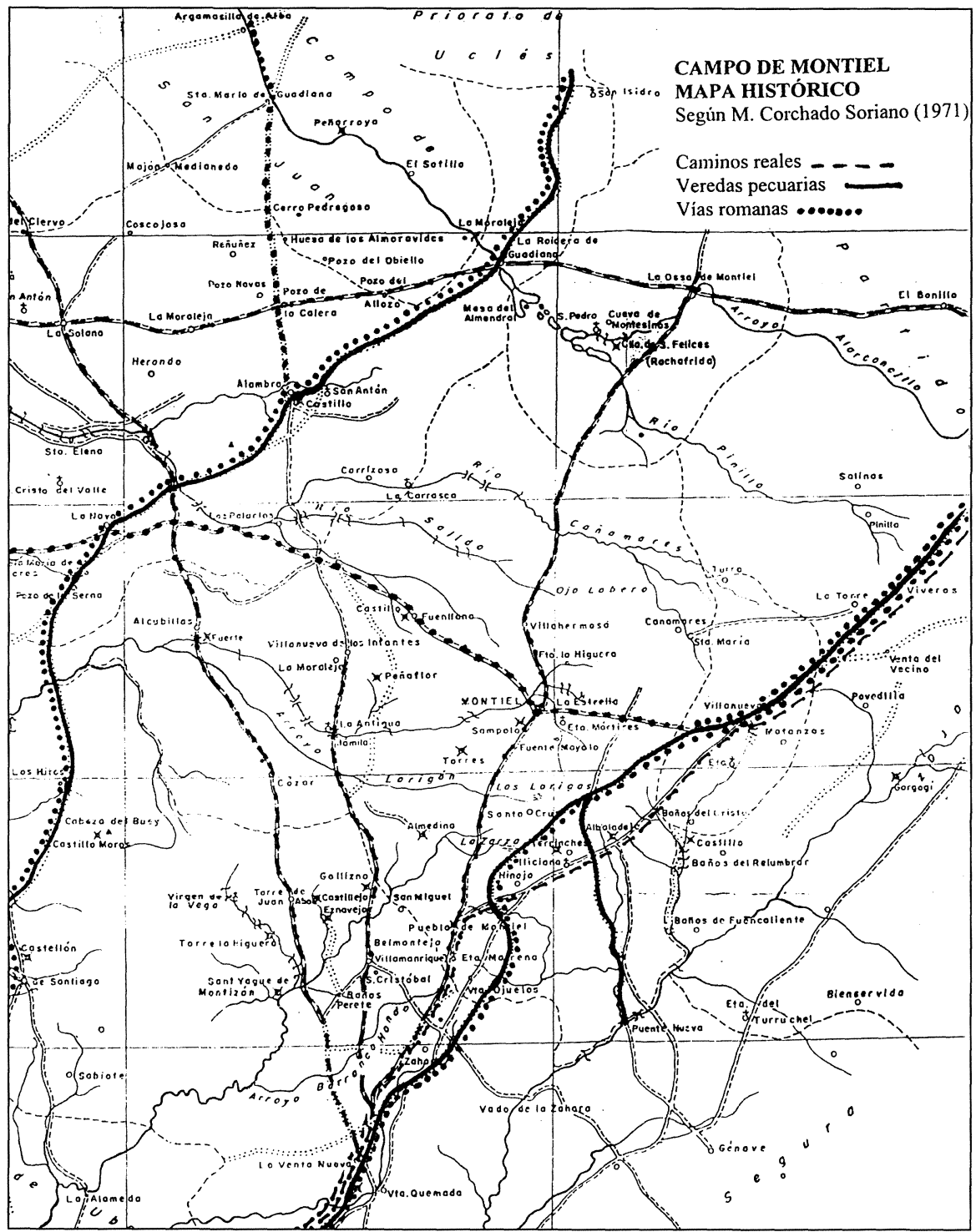

Figura 1.-Mapa histórico del Campo de Montiel según M. Corchado Soriano, Avance de un estudio geográfico-histórico del Campo de Montiel, Patronato José María Cuadrado del Consejo Superior de Investigaciones Científicas, 1971. 


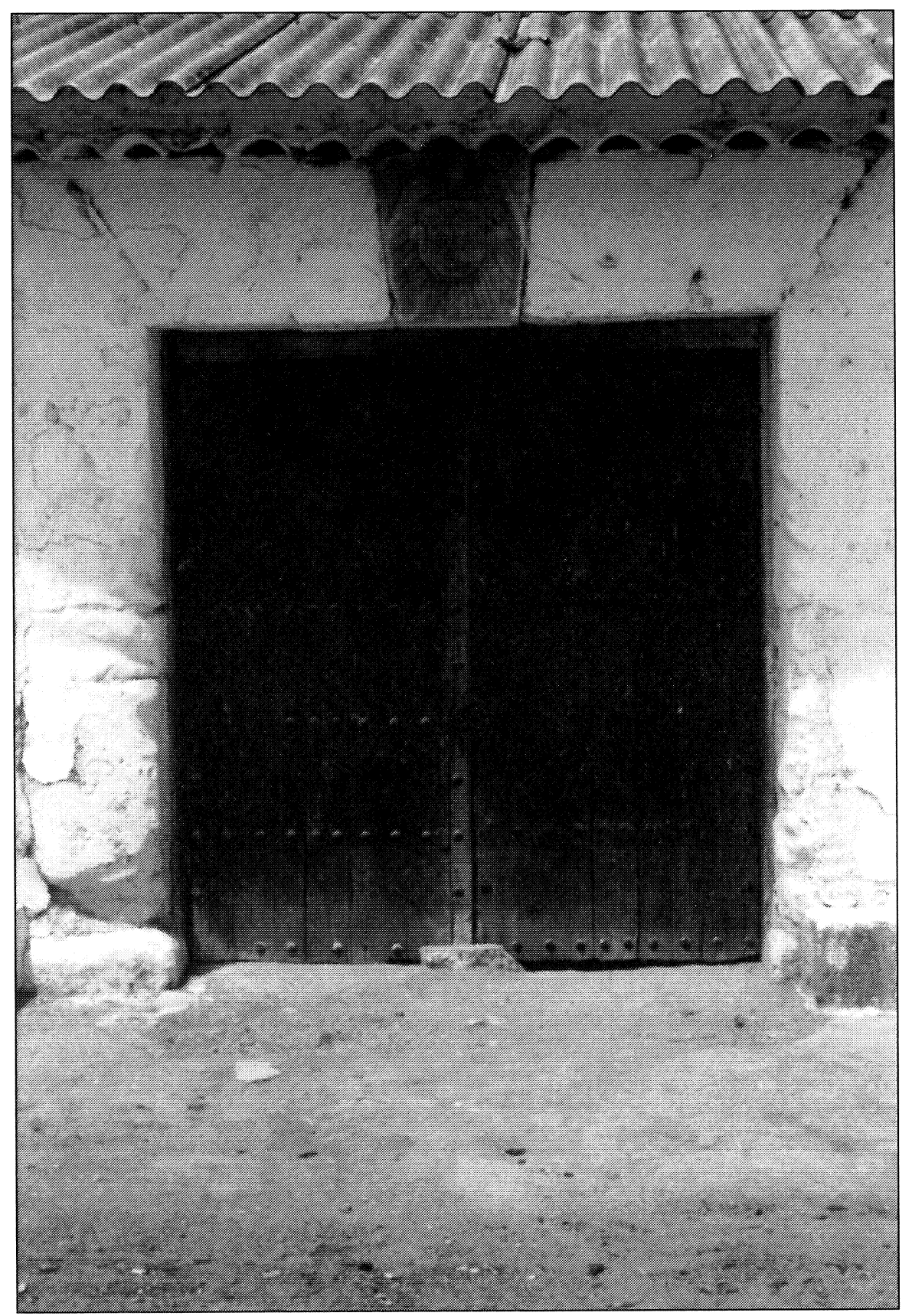

FIgURA 2.--Venta Nueva. Término municipal de Villamanrique. 


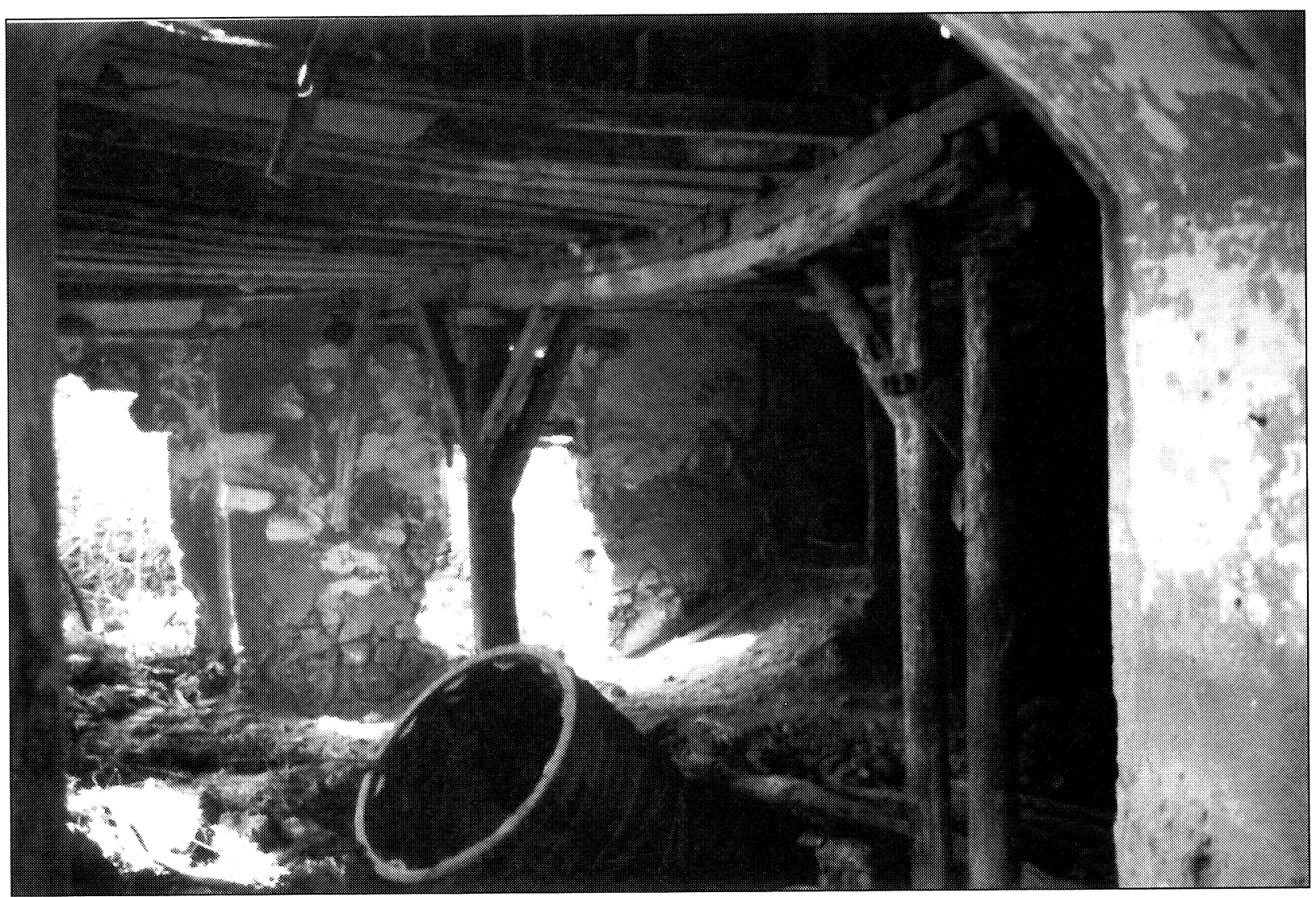

Figura 3.-Batán. Término municipal de Villanueva de la Fuente. 


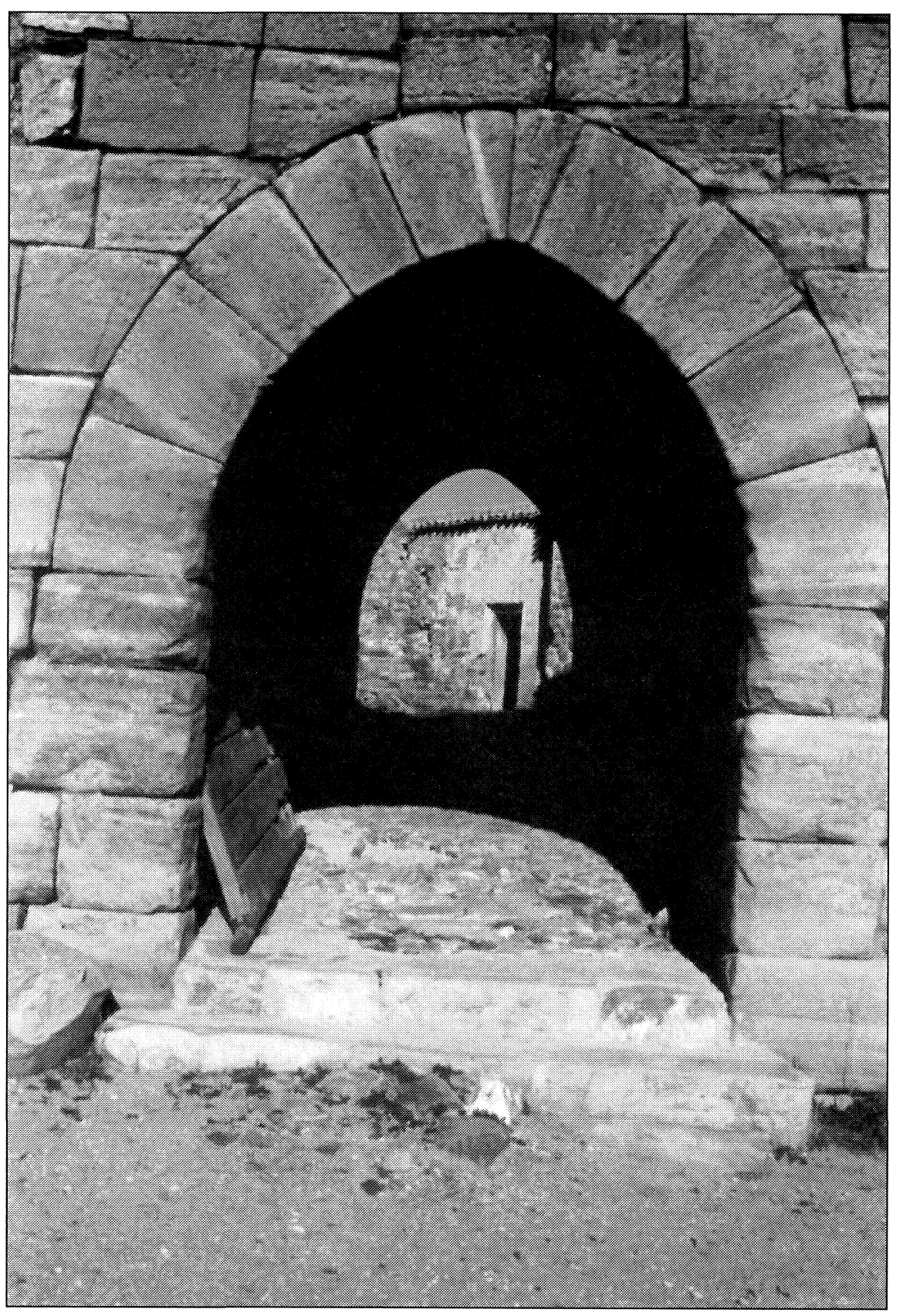

Figura 4.-Castillo de Montizón. Término municipal de Villamanrique. 


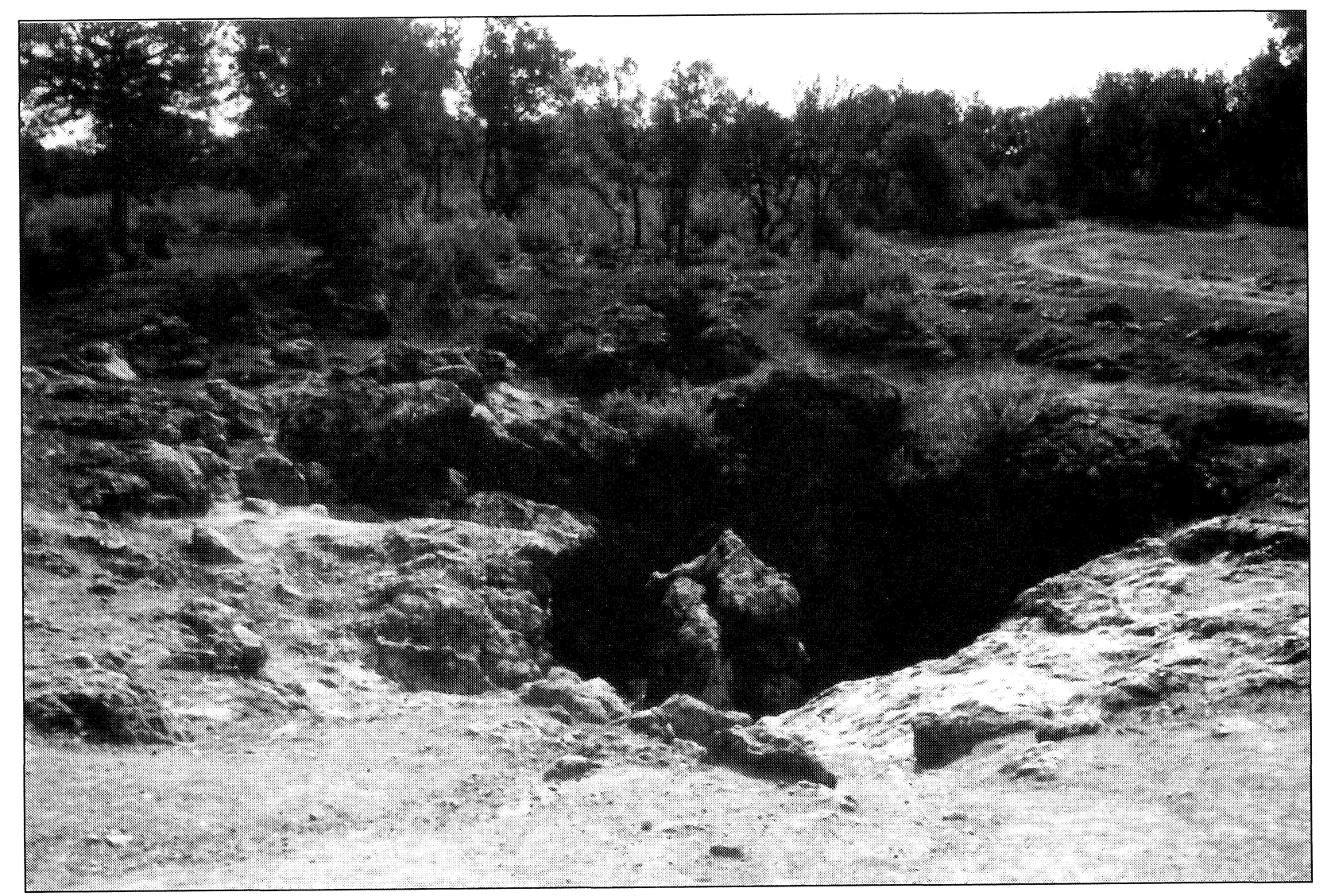

Figura 5.-Cueva de Montesinos. Término municipal de Ossa de Montiel. 


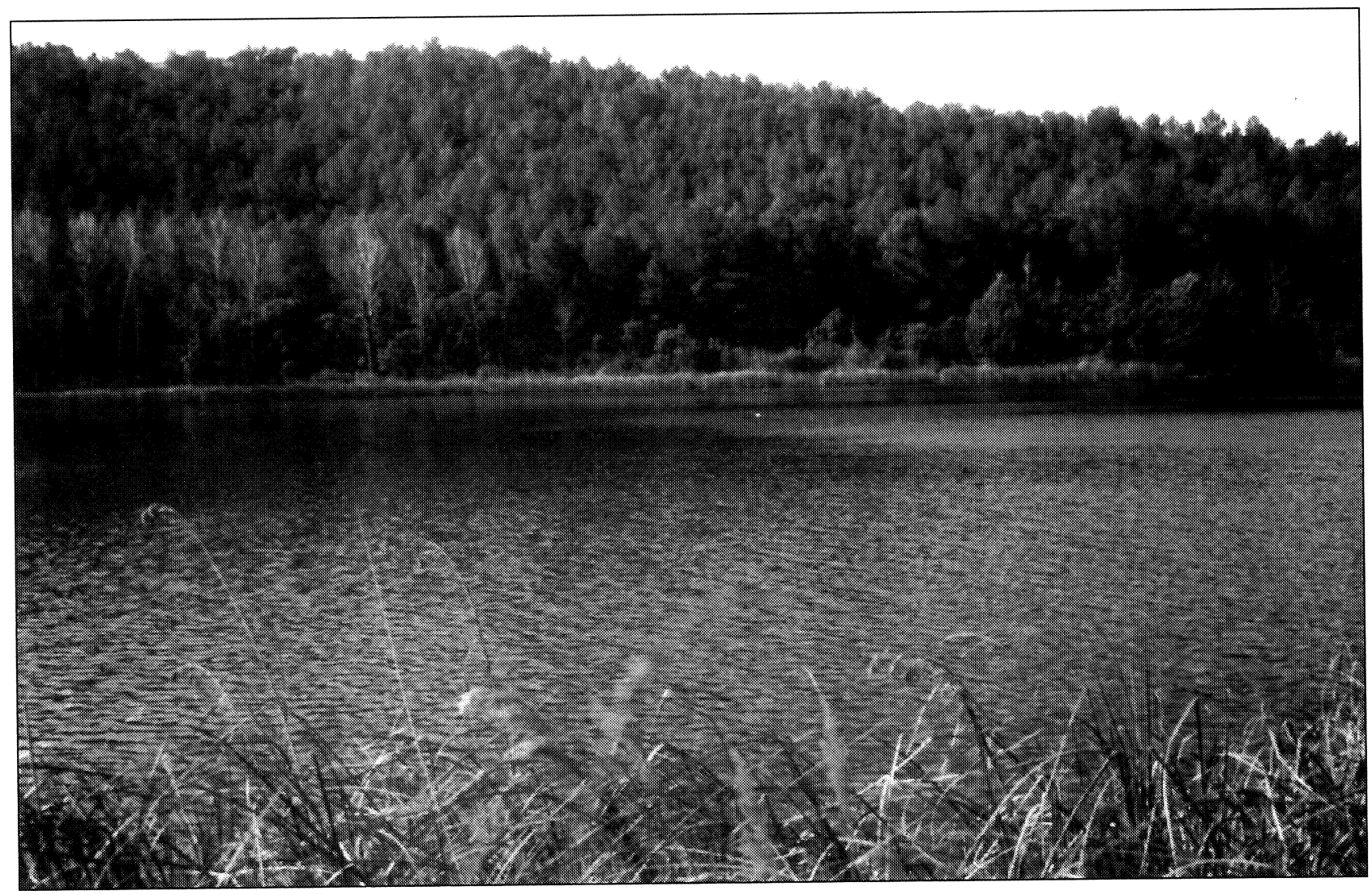

FigURA 6.-Lagunas de Ruidera. 


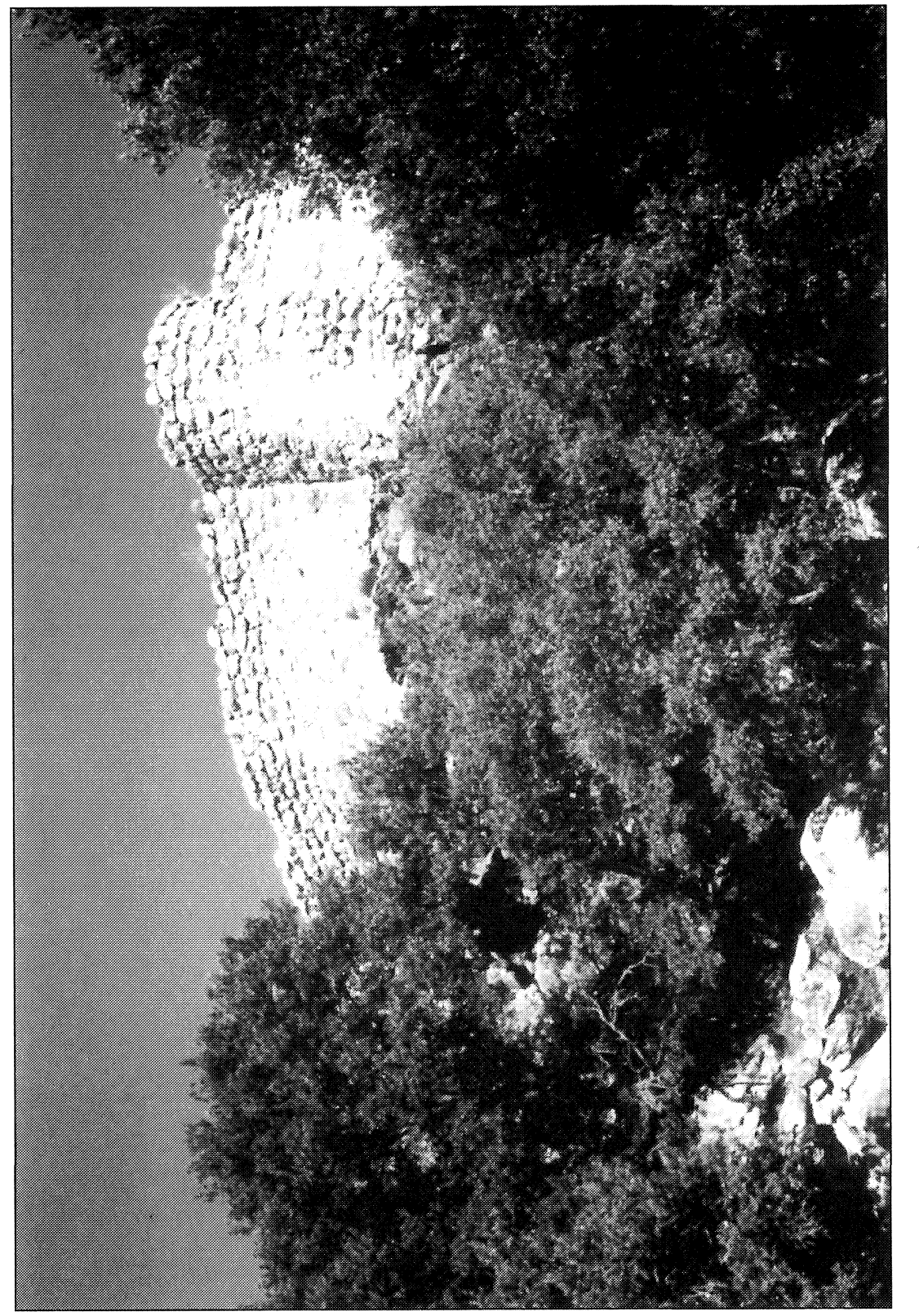

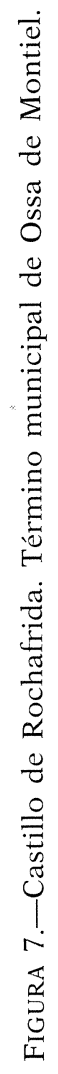

